\title{
Simulation of New Charge Summing and Hit Allocation Algorithm
}

\author{
Jakub Jirsa* \\ Faculty of Nuclear Sciences and Physical Engineering Czech Technical University in Prague, \\ Czech Republic \\ Faculty of Electrical Engineering Czech Technical University in Prague, Czech Republic \\ E-mail: jakub.jirsa@fjfi.cvut.cz
}

\section{Miroslav Havranek}

Faculty of Nuclear Sciences and Physical Engineering Czech Technical University in Prague,

Czech Republic

E-mail: miroslav.havranekefjei.cvut.cz

\section{Jiri Jakovenko}

Faculty of Electrical Engineering Czech Technical University in Prague, Czech Republic

E-mail: jakovenkefel.cvut.cz

\begin{abstract}
Modern VLSI technology allows the development of new class X-ray imaging detectors capableof capturing an image in various energy ranges in one shot. Such spectroscopic imaging detectorshave a high demand for the spatial and energy resolution of individual photons. With decreas-ing size of pixels, the charge cloud generated by the primary photon interaction, and in high-Zmaterials also by the fluorescent photon interaction, is shared across several pixels. That limitsboth spatial and spectroscopy resolution. This paper presents a novel charge summing algorithmcalled Winner-Master-Slave and describes the functionality, implementation, and simulation ofthe proposed algorithm in Verilog-AMS.
\end{abstract}

Topical Workshop on Electronics for Particle Physics TWEPP2019

2-6 September 2019

Santiago de Compostela - Spain

${ }^{*}$ Speaker. 


\section{Introduction}

Pixel detectors are commonly used in particle physics, medical imaging, and space science. The research nowadays tends to miniaturize the pixel size to increase the spatial resolution of pixel detectors. Each incident photon that interacts with detector material generates a charge cloud in the detector. In detectors which are made from high- $Z$ materials, the probability of emission of secondary fluorescence photon is increased. With decreasing pixels size, the charge cloud generated by the primary interaction and the subsequent fluorescent photons is shared across several pixels. That leads to the wrong allocation of primary interaction which decreases the spatial resolution of the detector. Fluorescent photons also degenerate the spectroscopic capabilities of detectors [1].

Several charge sharing compensation algorithms were developed and implemented [2, 3]. Most of the algorithms sum charge from four neighbouring pixels. This prevents the possibility of charge collection from more considerable distance which is generated by fluorescent photons.

This work presents a new fully asynchronous algorithm called Winner-Master-Slave (WMS), which deals with charge sharing effects as well as with fluorescent photons. WMS is designed to work fully asynchronous and it is capable of charge summing from 25 pixels (cluster size $5 \times 5$ pixels), which improves spectroscopic properties of the detector.

\section{WMS Algorithm}

\subsection{Principles of operation}

Every interaction of a particle with the detector material, which generates a charge cloud is called an event. Pixels with collected charge exceeding a specifically set threshold level are called active pixels. Whenever an event occurs, WMS automatically creates a cluster, sums the total charge from the active pixels in the cluster and then allocates the event to the pixel with the highest charge deposition. The algorithm consists of three types of pixels (Winner, Master and Slave) and uses a three-step procedure:

1. Select winner pixel: Pixel with the highest charge is selected as a winner.

2. Create cluster: Winner requests neighboring pixels for cluster creation.

3. Sum cluster charge: Winner sums charge from all active pixels in cluster and store its value.

The selection of the winner pixel is made right after an event occurs Figure 1 (a). Winner pixel is the active pixel with the highest charge deposition Figure 1 (b). When the winner pixel is selected, it requests its neighbouring pixels to become masters forming a $3 \times 3$ cluster depicted in Figure 1 (c). In the next step, all masters request their neighbouring pixels to join them into the cluster as slaves (Figure 1 (d)) and finalize phase two of cluster creation.

After cluster creation, the algorithm move in the summing phase. All active pixels which are assigned to the cluster are connected to the common current bus. Each pixel generates a current which is proportional to its deposited charge. The winner pixel is responsible for current summing and analog to digital conversion. Formation of a cluster is fast enough, thus it does not limit the maximum hit rate which is limited only by analog front-end electronics. 


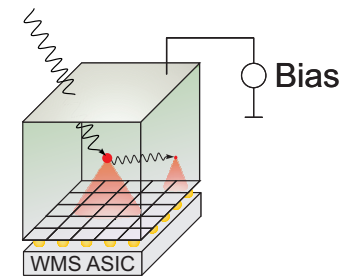

(a)

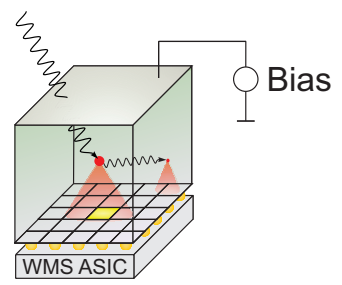

(b)

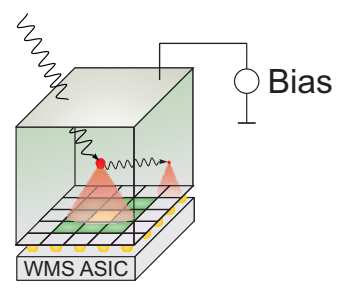

(c)

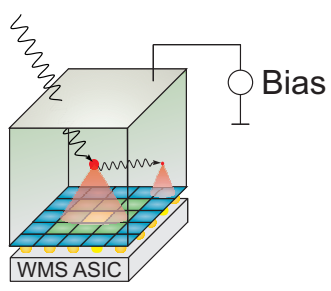

(d)

Figure 1: (a) hit with fluorescent photon, (b) Winner pixel selection - yellow, (c) Master pixel selection green, (d) Slave pixel selection - blue.

\subsection{Winner selection}

Pixel activation is related to the amount of charge. The higher is charge accumulation in a pixel, the faster is the process of pixel activation. Pixel with the highest charge is activated first. The winner pixel is selected based on arbitration between eight neighbouring pixels. For arbitration, standard asynchronous arbiters are placed between all neighbouring pixels. Arbiter decides between two requests, responding by grant signal to request which comes first. If neighbouring pixels are activated at the same time (i.e. both have the same amount of charge), arbiters are set to prefer one of them. This prevents the occurrence of two winners next to each other. Pixel is selected as the winner only if it is activated and wins all arbitrations between its neighbours.

\subsection{Cluster creation}

Pixels intercommunication consists of three types of buses: master bus, slave bus, and the current bus. Master and slave buses are used for cluster formation. Meanwhile, the current bus is used for charge summing. When the winner pixel is selected, it sends to its neighbouring pixels request to join into the cluster $(3 \times 3)$ as master pixels. This request is sent through the master bus. Pixel that receives master request joins the cluster as a mater and saves the position of winner pixel. The location of the winner pixel plays an essential role in sending slave requests and charge summing.

Pixels that have joined the cluster as masters send slave requests based on the position of winner pixel Figure 2. Master pixels that are located in one of four world directions - North $(\mathrm{N})$, West (W), South (S), East (E) from the winner pixel send slave request in the opposite direction than the location of winner pixel. Master pixels that are located at the corners of the cluster $3 \times 3$ (Northwest (NW), Southwest (SW), Southeast (SE) and Northeast (NE)) send three slave requests to their neighbouring pixels. 


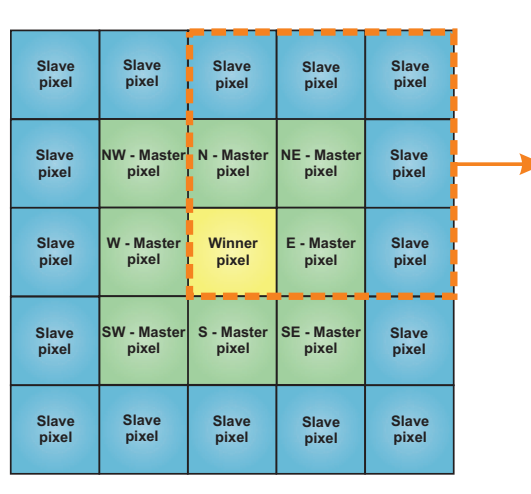

(a)

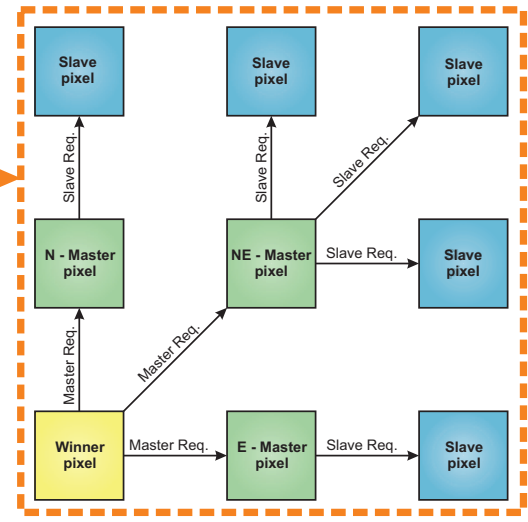

(b)

Figure 2: (a) formed cluster, (b) communication between northeast neighbouring pixels

In case of multiple hits which are located close to each other several situations can occur: When hits are located at neighbouring pixels the situation is resolved by arbitration, which leads to selection of one winner. When hits are located over the distance of one pixel, the rule of "slave takes winner" is applied which leads to the creation of one cluster with one winner. When hits are located over the distance of two pixels the rule of "master draws with master" is applied and smaller clusters are created with master pixels at the neighbouring sides. Finally, when hits are located over the distance of three pixels winners compete for slave pixels. In all described situations each pixel belongs to just one cluster.

\subsection{Charge summing}

Summing phase starts when the cluster is formed. All pixels which are part of a cluster are connected through switches to the common current bus. The winner is responsible for grounding the bus and summing currents. The current flow generated by each pixel is proportional to the amount of charge in it. For simulation purposes, the winner pixel grounds the current bus with termination resistance. The total charge corresponds to the voltage drop across the resistance. The winner pixel then samples the voltage and releases pixels in the cluster.

\section{Simulation results}

\subsection{Simulation methodology}

The algorithm was implemented in Verilog-AMS and simulated with AMS simulator in Cadence Virtuoso environment. The size of the pixel matrix was set to 10x10. The charge clouds with Gaussian distribution were randomly placed over the pixel matrix. The maximum size of the charge cloud was set to $5 \times 5$. The total number of charge clouds in one frame was set to three. Total charge deposition in one cluster was 27000e. To simulate the effect of fluorescent photons nonzero probability of generation charge cloud of size $1 \mathrm{x} 1$ with $6000 \mathrm{e}$ was added. The fluorescent photon can travel anywhere within 2 pixels aside from primary interaction.

WMS resolves all single-photon hits with charge sharing effect with cluster size up to $5 \times 5$ pixels. It also resolves events with fluorescent photons Figure 3. WMS reconstructs the total 
energy deposit by the incident photons and successfully allocates a summed charge to the pixel where the primary interaction was.
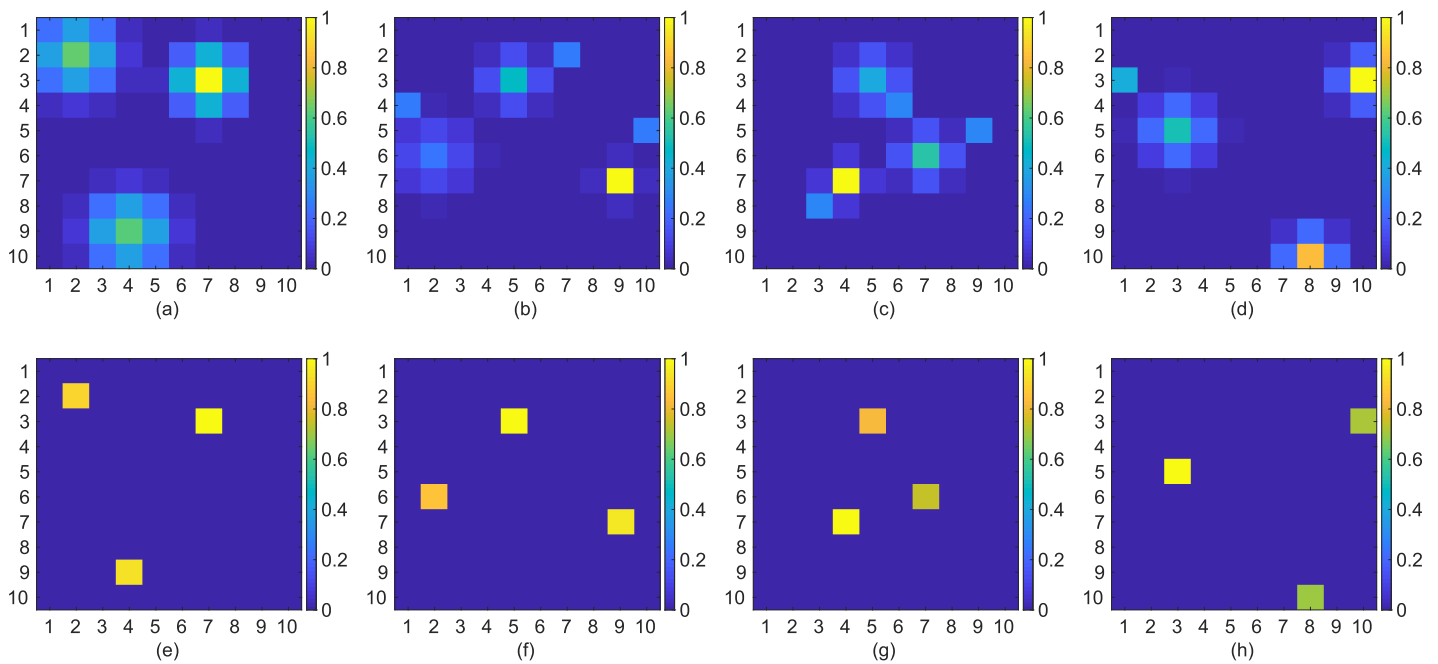

Figure 3: Three separate hits (a) - (d) and their resolution and allocation (e) - (h).

\section{Conclusion}

This paper presented a novel charge summing algorithm. WMS algorithm was designed in Verilog-AMS and simulated with AMS simulator in Cadence Virtuoso environment. The algorithm has been systematically tested using randomly generated clusters. The simulation outcome proved that WMS algorithm solves several problems of existing solutions and is suitable for implementation in a front-end ASIC.

\section{Acknowledgment}

This work was supported by Czech Ministry of Education, Youth and Sports of the Czech Republic under project number LM2015049, CZ.02.1.01/0.0/0.0/16-013/0001677, LM2015058, Centre of Advanced Applied Sciences funded by grant CZ.02.1.01/0.0/0.0/16-019/0000778, cofinanced by European Union and Czech Grant Agency of the Czech Technical University in Prague, grant No. SGS17/188/OHK3/3T/13.

\section{References}

[1] D. Pennicard and H. Graafsma, Simulated performance of high-Z detectors with Medipix3 readout, Journal of Instrumentation 6 (2011) P06007.

[2] A. Krzyzanowska, G. W. Deptuch, P. Maj, P. Grybos and R. Szczygiel, Characterization of the Photon Counting CHASE Jr., Chip Built in a 40-nm CMOS Process with a Charge Sharing Correction Algorithm Using a Collimated X-Ray Beam, IEEE Transactions on Nuclear Science 64 (2017) 2561.

[3] P. Otfinowski, G. W. Deptuch and P. Maj, Asynchronous Approximation of a Center of Gravity for Pixel Detectors' Readout Circuits, IEEE Journal of Solid-State Circuits 53 (2018) 1550. 